\title{
Risky Business: Sexual and Asexual Reproduction in Variable Environments
}

\author{
Arthur J. Robson*, Carl T. Bergstrom $†+$ and Jonathan K. Pritchard $†$ \\ *Department of Economics, University of Western Ontario, London, Ontario, \\ Canada N6A 5C2 and †Department of Biological Sciences, Stanford University, \\ Stanford, CA 94305, U.S.A.
}

(Received on 24 November 1997, Accepted in revised form on 23 December 1998)

\begin{abstract}
Patterns of reproductive uncertainty can have an important influence on population dynamics. There is a crucial distinction between what we describe here as aggregate uncertainty (in which reproductive output in each generation is correlated among the individuals in a population) and idiosyncratic risk (in which reproductive output is independent across individuals). All else being equal, populations experiencing idiosyncratic risk enjoy a higher asymptotic growth rate than do those experiencing aggregate uncertainty. Therefore individuals in populations of the former type will have a competitive advantage over individuals in populations of the latter type. Applying this distinction to models of randomly fluctuating environments, we point out that genetic variation among offspring can serve to reduce aggregate uncertainty, transforming it into a more idiosyncratic form of risk. We show tht this transformation underlies the dynamics observed in several previous models of the role of outcrossing in the evolution of sex.
\end{abstract}

(C) 1999 Academic Press

\section{Introduction}

The fitness concept in theoretical biology is commonly used to predict the results of competition among different types of individuals. In making such predictions, one cannot always rely on expected fitness in a single generation; rather, it has long been known that one must consider the degree of variation in fitness over time.

$\$$ Present address: Department of Biology, 1510 Clifton Road, Emory University, Atlanta, GA 30322, U.S.A.

$\S$ Author to whom correspondence should be addressed. Present address: Department of Statistics, 1 South Parks Road, Oxford University, Oxford OX1 3TJ, U.K.

E-mail: pritch@stats.ox.ac.uk
Dempster (1955) was perhaps the first to demonstrate that long-term success under fluctuating environmental conditions is determined by the geometric rather than arithmetic mean fitness over time; many subsequent papers (reviewed by Gillespie, 1991) have refined these results. Lewontin \& Cohen (1969) give an example of a fitness distribution for which the expected population size grows indefinitely while the probability of population extinction goes to one.

Indeed, population growth depends on more than simply the moments of the fitness distribution; the dynamics of populations experiencing fluctuations in fitness will depend critically on the correlations in fitness among individuals (Seger \& Brockmann, 1987). For this reason, it is crucial to distinguish between two

C) 1999 Academic Press 
different types of uncertainty in reproductive output which may be faced by individuals. We borrow terminology from the economics literature (e.g. Kreps, 1990), defining the first of these types, idiosyncratic risk, to be risk which is independent of that faced by other individuals. We contrast this with a second type which we call aggregate uncertainty: risk which is perfectly correlated among individuals (cf. Yoshimura \& Clark, 1991). For example, herbivory could create primarily idiosyncratic risk for plants in a lightly grazed field, whereas drought would affect all individuals or none, and thus act as a form of aggregate uncertainty. Even though an individual's immediate distribution of offspring number might not be affected by whether a particular source of risk is idiosyncratic or aggregate, the nature of the risk will systematically affect the distribution of offspring number in all subsequent generations. Notice that here spatial fluctuations in the environment (in the form of herbivory) act as a source of idiosyncratic risk, whereas temporal fluctuations (in the form of climatic change) act as a source of aggregate risk. This is a common feature of ecological models.

The distribution of genetic variation among offspring, which depends in part on patterns of reproduction, may affect the levels of idiosyncratic and aggregate risk faced by members of a population. In this paper we explore the role that genetic variation can play in reducing the aggregate risk to a population. In particular, we argue that environmental factors which are sources of aggregate risk to asexuals may to some extent constitute idiosyncratic risk to sexually reproducing individuals. Consideration of the different impacts of idiosyncratic risk and aggregate uncertainty on sexuals and asexuals thus contributes to a deeper understanding of why sex, or more precisely, outcrossing, is maintained through evolutionary time. (The models examined in this paper are all single-locus models, and as such the dynamics are affected by outcrossing but not recombination).

We begin with a brief review of relevant results on population dynamics in randomly fluctuating environments. We then turn our attention to genetic models in randomly fluctuating environments. We point out that, in such models, genetic variation among offspring can reduce the level of aggregate uncertainty, effectively making the risk more idiosyncratic. As illustrations, we consider the random environment models of Weinshall (1986), Roughgarden (1991), and Hines \& Moore (1981) which were originally proposed because they favor outcrossing over asexual reproduction. We show that the advantage experienced by outcrossing types in those models stems from the fact that their offspring generally experience lower aggregate uncertainty than do those of their asexual counterparts.

\subsection{POPULATION GROWTH WITH AGGREGATE UNCERTAINTY AND IDIOSYNCRATIC RISK}

There are a number of ways in which we might compare the long-term success of populations subject to environmental uncertainty. Let us consider which of these will be most useful as a measure of density-independent population growth. In models of this kind, the growth rate is sometimes expressed as the root of expected population size $\left(E\left[z_{T}\right]^{(1 / T)}\right.$, where $z_{T}$ is the population size at time $T$. This represents an extension to the random environment case of the measure proposed by Dempster (1955) and Haldane \& Jayakar (1963) who studied fixed environmental sequences. However, when comparing the growth rates of two populations in a random sequence of environments, this measure can be misleading, because it is often dominated by the extreme right-hand tail of the distribution. That is, the expectation of the population size is often vastly inflated by rare events in which the population is wildly successful. When trying to predict the outcome of competition between populations, we are concerned not so much with the margin of victory as with the probability of victory. It is more useful in this context to compare the values of $E\left[\left(z_{T}\right)(1 / T)\right]$ (Gillespie, 1973; Tuljapurkar \& Orzack, 1980; Gillespie, 1991; Haccou \& Iwasa, 1995). This measure is determined primarily by the most common values of the distribution of population sizes. In the examples below, $z_{T}^{(1 / T)}$ converges to a constant, so that taking the expectation is unnecessary. It is then equivalent to consider the measure $(1 / T) \ln \left(z_{T}\right)$, which also converges with probability 1 to a constant.

In the following example, we will refer to the quantity $\left(E\left[z_{T}\right]\right)^{(1 / T)}$ as the "geometric mean 
expected growth rate" and to $E\left[\left(z_{T}\right)^{(1 / T)}\right]$ as the "average long-term growth rate." When considering the $\log$ form of the latter measure, $\left.\ln z_{T} / T\right]$, we will use the label "long-term log growth rate". We now describe two populations with differing patterns of risk, and show both the distinction between these two growth measures and the distinction between different forms of risk.

Consider an environment with two states, I and II, each occurring independently in each generation $T=1,2, \ldots$ with probability $1 / 2$. Individuals denoted "Type A" produce one offspring in state I and two offspring in state II. There is no density dependence. Let $z_{T}(A)$ be the number of individuals of type A at time $T$. Clearly, $z_{T}(A) \rightarrow \infty$ with probability 1 , as $T \rightarrow \infty$. To compute the long-term log growth rate of type $\mathrm{A}$, we note that $z_{T}(A)=2^{n(T)}$, where $n(T) \in 0, \ldots, T$ is the number of times that state II is observed in $T$ periods. The strong law of large numbers implies that $n(T) / T \rightarrow 1 / 2$ with probability 1 as $T \rightarrow \infty$. Hence $1 / T$ $\log \left(z_{T}(A)\right) \rightarrow \sqrt{2}$ with probability 1 as $T \rightarrow \infty$.

Individuals denoted "Type B" inhabit the same environment, but are not affected by the random fluctuations. Instead, each individual procuces one or two offspring with probability $1 / 2$ in both environments. Note that the immediate offspring distribution of type $\mathrm{B}$ individuals is identical to that of type $\mathrm{A}$ individuals. Defining $z_{T}(B)$ as the number of individuals of type $\mathrm{B}$ at time $T$, we have again that $z_{T}(B) \rightarrow \infty$ with probability 1 as $T \rightarrow \infty$. The theory of branching processes implies that $z_{T}(B) /(3 / 2)^{T}$ converges to a strictly positive random variable $W$, with probability 1 , as $T \rightarrow \infty$ (see Athreya \& Ney, 1972, Theorems $1-2)$. This implies that

$$
\frac{1}{T} \ln \left(z_{T}(B)\right) \rightarrow \ln \left(\frac{3}{2}\right)
$$

with probability 1 , as $T \rightarrow \infty$, so that the constant $\ln (3 / 2)$ is the long-term log growth rate of Type B.

This measure of the growth rate reveals the difference between the long-term consequences of idiosyncratic and aggregate risk. That is, it follows that

$$
\frac{1}{T} \ln \left(\frac{z_{T}(B)}{z_{T}(A)}\right) \rightarrow \ln \left(\frac{3}{2 \sqrt{2}}\right)>0
$$

with probability 1 , as $T \rightarrow \infty$, so that

$$
\frac{z_{T}(B)}{z_{T}(A)} \rightarrow \infty
$$

with probability 1 . Thus Type B ultimately dominates the population in this appropriately compelling sense.

Despite this result, notice that the expected sizes of the two populations are always equal, both being $(3 / 2)^{T}$ in period $T$. The geometric mean expected growth rate is $3 / 2$ in both cases and therefore this measure fails to distinguish between the two populations. Although the number of occurrences of state II in each environmental sequence is symmetrically distributed, the size of the Type A population has a skewed distribution, with its right-hand tail contributing disproportionately to the expectation. This occurs because the size of the Type A population is exponential in the number of occurrences of state II and thus the median of the Type A distribution falls increasingly below the mean as $T$ increases. Remarkably, in the limit, all of the probability mass of the Type A distribution falls below its own mean and, indeed, also below all of the probability mass of the type $\mathrm{B}$ distribution.

The intuition for the distinction between aggregate and idiosyncratic risk in this example can be recast as follows. In the case of idiosyncratic risk, as the population size becomes large, the growth rate in each period converges to $3 / 2$, by the law of large numbers. It follows that the average long-term growth rate then also converges to $3 / 2$. By contrast, in the case of aggregate uncertainty, the growth rate is always stochastic, being either 2 or 1 with equal probability. As $T$ goes to infinity, the fraction of 2 s observed up to time $T$ converges to $1 / 2$ with probability 1 by the strong law of large numbers. It follows that the average long-term growth rate converges to $\sqrt{2}$.

While this example includes no density-dependent effects, the point illustrated would survive 
the incorporation of environmental limitations on population size. From this simple example, it becomes clear that idiosyncratic risk and aggregate uncertainty can have dramatically different evolutionary consequences. This theme is central to our paper.

We now examine a general model of population growth in the presence of environmental uncertainty. Consider an asexual population whose generations are discrete and non-overlapping, represented by a branching process. An environment is selected by a random process and, conditional on this environment, each individual produces some number of offspring drawn from an i.i.d. distribution (e.g. see Athreya \& Ney, 1972). Again there is no density dependence in this system. This model incorporates both aggregate uncertainty of the population due to shared environment, and idiosyncratic risk experienced separately by each individual.

There is a fixed, finite set $S$ of possible environmental outcomes, designated $E_{1}, E_{2}, \ldots$, $E_{S}$. Let the stochastic process generating the environments in different generations be stationary and ergodic. Suppose the stationary frequencies of these outcomes are $\pi_{1}, \pi_{2}, \ldots, \pi_{S}$, respectively, where $\pi_{s} \geqslant 0$ for all $s$, and $\Sigma_{s=1}^{S}$ $\pi_{s}=1$. At time $t$, the random variable $\xi_{t}$ is the current state of the environment.

The number of offspring produced by each individual at time $T$ is a random variable, the distribution of which is determined by the realization of $\xi_{T}$. Let $p_{j}\left(E_{s}\right)$ be the probability of exactly $j$ offspring surviving in environment $E_{s}$, where $s \in\{1, \ldots, S\}$. Conditional on $E_{s}$, the offspring distribution is given by $p_{0}\left(E_{s}\right), \ldots$, $p_{B}\left(E_{s}\right) \geqslant 0$, where $\Sigma_{j=0}^{B} p_{j}\left(E_{s}\right)=1$, and $B$ is the largest possible number of offspring. The reproduction of each individual is independent of that of other individuals conditional on the environment. Define the expected number of offspring generated by an individual in environment $E_{s}$ as $m\left(E_{s}\right)=\sum_{j=0}^{B} j p_{j}\left(E_{s}\right)$.

Under such a model, it can be shown that the long-term log growth rate, $\rho$ is given by

$$
\rho=\sum_{s=1}^{S} \pi_{s} \ln \left(m\left(E_{s}\right)\right)
$$

Similar expressions have been obtained by Gillespie (1973), Cohen (1993), Haccou \& Iwasa (1995), Sasaki \& Ellner (1995), and Robson (1996). The value of $\rho$ is directly related to the probability of extinction. In the subcritical case where $\rho<0$ the probability of extinction is 1 . This is also true in the critical case where $\rho=0$ (subject to a mild non-degeneracy condition). In the supercritical case where $\rho>0$, the probability of extinction is strictly less than one (see Athreya \& Ney, 1972, 1a and 1b; Theorem 4). It can be shown (Robson, 1996) that these results hold even starting from a small initial population size.

The crucial point here is that the long-term log growth rate $\rho$ separates idiosyncratic risk (realized fitness, depending on $E_{s}$ ) from aggregate uncertainty $\left(\pi_{s}\right)$. Since the logarithm is a concave function, population growth is more rapid when there is an equal risk in all environments, than when some environments are better than others (provided that the average itself remains the same). In other words, population growth is greater when individuals face risks independent of each other than when risks are shared (see also Seger \& Brockmann, 1987 in this context).

\section{The Role of Genetic Variation in a Random Environment}

In the previous section, we discussed a simple model which highlights the way that the distribution of risk can impact population growth. That model was concerned with monomorphic population dynamics and ignored the possibility of genetically different and sexually interacting individuals. In this paper, we argue that outcrossing affects the distribution of genetic variation and that the presence of genetic variation in turn impacts the distribution of risk in a population, making an outcrossing population seem more idiosyncratic than an otherwise equivalent non-outcrossing population.

Consider a sexual population of an annual plant. In each year, the environment might be unpredictable: perhaps warm and wet, perhaps cool and dry. If an individual produces offspring which are all genetically uniform, all individuals are affected similarly by the environmental 
conditions and therefore this constitutes purely aggregate risk. If, in contrast, a parent produces offspring with a mixture of genotypes, each of which is best-suited to a different environment, different individuals are affected differently by the environmental conditions and thus some of the aggregate uncertainty is replaced by idiosyncratic risk.

It follows from our results in the previous section that a population with little genetic variation at the loci under fluctuating selection is highly susceptible to extinction under such a scenario. If only one or a few distinct genotypes are present, the effects of aggregate uncertainty on population dynamics become very important. In order to prevent uncertainty from becoming fully aggregate, it is essential that the population maintain sufficient levels of genetic variation. As such, it will be important to determine whether a population can maintain these levels of variation over an extended period of time.

We now examine these issues using a genetic model of evolution in randomly fluctuating environments (Weinshall, 1986). We investigate genetic variation and aggregate uncertainty in that particular model, and show that an outcrossing population is able to maintain enough variation to allow indefinite population survival, despite the fact, as we show later, that a selfing or genetically monomorphic population could not survive in the same environment.

We consider the model of Weinshall (1986, also discussed in Eshel \& Weinshall, 1987 and Weinshall \& Eshel, 1987), restricting our attention at first to a population of randomly mating sexual diploids. In this model, the population experiences one of three randomly chosen environments, labelled I, II, or III, in each generation. (Weinshall also considers a two-environment model; there, outcrossing is not favored.) This environment is chosen independently at each date with a probability of $1 / 3$ for each possibility. We consider a single selected locus with three possible alleles, A, B, and C. Each allele is advantageous in a different environment: alleles $\mathrm{A}, \mathrm{B}$, and $\mathrm{C}$ being favored in environments I, II and III, respectively. There is no mutation. An individual with no copies of the allele favored by the current environment has zero fitness. The fitness table is given in Table 1.
Weinshall's model was originally framed in a density-dependent setting, with a fixed population size. However, here we consider the "hard selection" case, in which the fitnesses shown in Table 1 are absolute, and the population size is allowed to change without density dependence.

The density-independent setting, though not strictly realistic, has the important advantage that it allows us to address the question of long-term population survival. It is not entirely convincing to prove that sexuals can outcompete asexuals in a soft selection model, if the long-term survival of the sexuals is guaranteed only by the assumption of a fixed population size.

One implicit aspect of this table deserves mention. The fitness values $x$ and $y$ reflect expected reproductive success conditional on the corresponding environment. For reasons similar to those discussed above, this is an appropriate measure of average fitness in a large population if the underlying conditional distribution of offspring is idiosyncratic. That is, each of a large number of individuals of a particular genotype enjoys reproductive success obtained by an independent draw from the same distribution conditional on the environment. This particular source of risk will not be considered further.

Suppose that the numbers of copies of alleles $\mathrm{A}, \mathrm{B}$ and $\mathrm{C}$ are initially $a, b$ and $c$, respectively. Denote by $a_{i}, b_{i}$ and $c_{i}$ the number of copies of each allele in the next generation, conditional on the occurrence of environment $i=\mathrm{I}$, II, or III. As a consequence of random mating, the population genotype frequencies are in Hardy-Weinberg proportions prior to selection.

TABLE 1

Genotype fitnesses in environments I, II, and III. Here $\mathrm{x} \geq \mathrm{y} \geq 0$. Notice that when $\mathrm{x}=\mathrm{y}$, the alleles are in effect dominant, and when $\mathrm{y}=0$, the alleles are recessive

\begin{tabular}{llll}
\hline & I & II & III \\
\hline AA & $x$ & 0 & 0 \\
AB & $y$ & $y$ & 0 \\
AC & $y$ & 0 & $y$ \\
BB & 0 & $x$ & 0 \\
BC & 0 & $y$ & $y$ \\
CC & 0 & 0 & $x$ \\
\hline
\end{tabular}




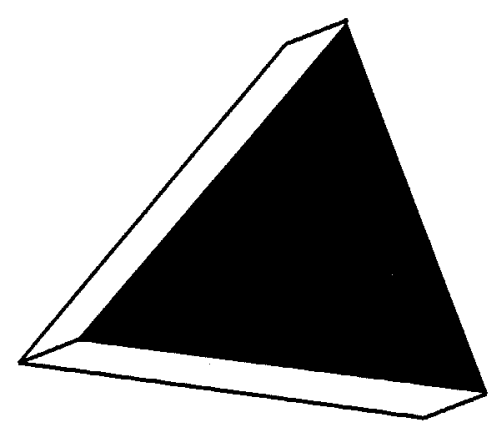

(a) Probability density at $\mathrm{t}=0$

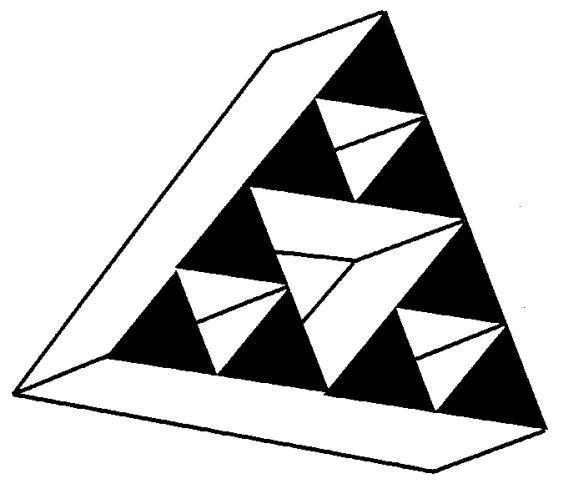

(c) Probability density at $\mathrm{t}=2$

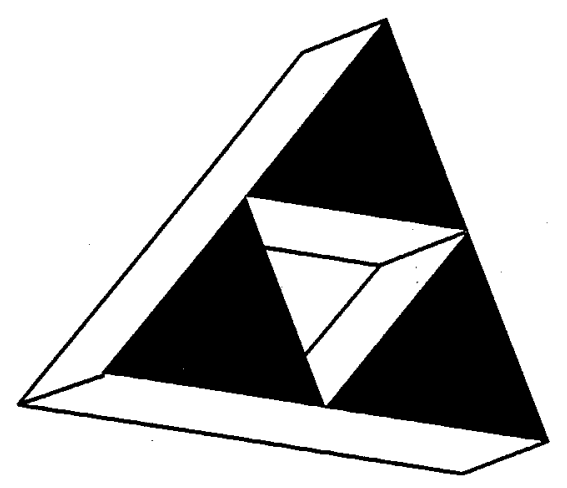

(b) Probability density at $\mathrm{t}=1$

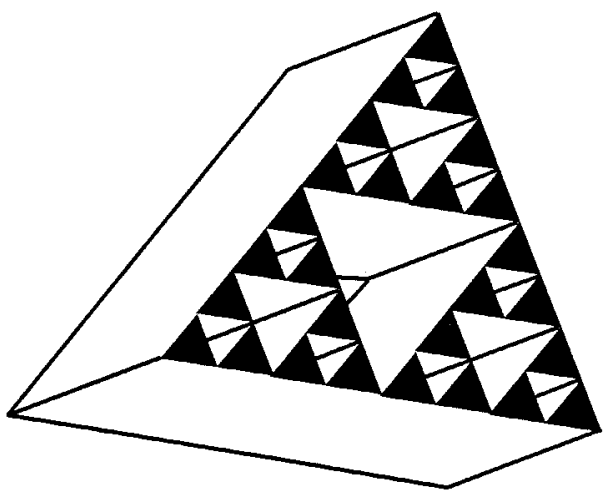

(d) Probability density at $\mathrm{t}=3$

FIG. 1. Starting with a uniform distribution of allele frequencies over the simplex at time $t=0$, application of the recursions for Weinshall's model with $x=2 y$ gives allele frequencies uniformly distributed over the $3^{t}$ shaded regions of the Sierpinski triangle at time $t$. Notice that, in each generation, the area of the simplex with positive probability density decreases to $3 / 4$ of its previous value and, correspondingly, the probability density at all non-zero points increases by $4 / 3$.

Defining $N=a+b+c$, the recursion for allele copy-number, conditional on the occurrence of environment $\mathrm{I}$, is

$$
\begin{gathered}
a_{I}=a(a x+b y+c y) / N \\
b_{I}=a b y / N \\
c_{I}=a c y / N
\end{gathered}
$$

Similar recursions hold for environments II and III. We begin the analysis by showing how the level of aggregate uncertainty depends on allele frequencies. Let $p, q$, and $r$ be the frequencies of alleles $\mathrm{A}, \mathrm{B}$, and $\mathrm{C}$, respectively (i.e. $\quad p=a / N, \quad q=b / N, \quad r=c / N \quad$ and $p+q+r=1)$. Then the expected fitness in environment $\mathrm{I}$ is

$$
x p^{2}+2 y(p q+p r) .
$$

Likewise, the expected fitnesses in environments II and III are $x q^{2}+2 y(q p+q r)$ and $x r^{2}+2 y(r p+r q)$, respectively. Thus, for example, in the special case where $x=2 y$, the expected population fitness is $x / 3$ for all values of $p, q$, and $r$, since each environment arises with probability $1 / 3$.

However, as argued previously, expected fitnesses can be misleading when we wish to predict long-term survival, or the outcome of competition between two types. In a genetic model such as Weinshall's, population growth in each generation depends not only on the environment, but also on allele frequencies. Hence, to understand the process of long-term growth in the Weinshall model, it will be necessary to know what happens to allele frequencies over time. 
In order to analyse the evolutionary trajectory of allele frequencies in this system, we make use of the two-dimensional simplex in Fig. 1(a). Each point within the simplex represents a possible set of the allele frequencies, $p, q$, and $r$, each of which is given by the distance from one edge. First, consider Recursion (4) with $x=2 y$. The application of this recursion, with equal probabilities of each environment, corresponds to the process of randomly selecting a vertex of the simplex, and moving half-way from the current position toward this vertex on a direct line. This procedure generates the allele frequencies in the next generation, and we repeat the procedure to generate the allele frequencies in subsequent generations.

If the probability density of the allele frequencies at time $t=0$ is given by a uniform distribution over the simplex, then at time $t=1$ after following this rule, the probability density of allele frequencies will be a uniform distribution over the region shaded in Fig. 1(b).
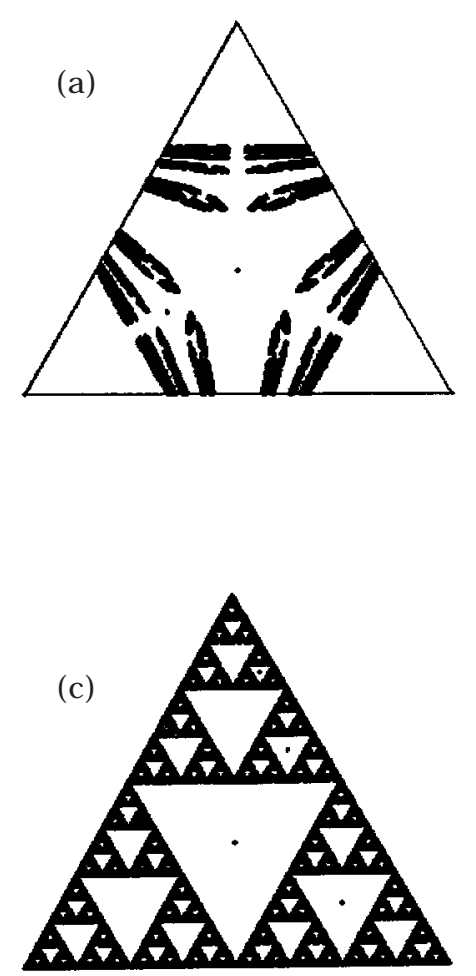

Continuing to apply the rule, the probability densities at times $t=2$ and $t=3$ will be uniform over the shaded regions in Fig. 1(c) and 1(d), respectively. The fractal structure representing these probability distributions is known as the Sierpinski Gasket (Mandelbrot, 1982). The allele frequencies at time $t$ are uniformly distributed over the Sierpinski triangle with $3^{t}$ distinct shaded regions, as is proven in Appendix A.

We can also examine the allele frequency distributions for other values of $x$ and $y$. Several examples are shown in Fig. 2.

It is evident that the system spends essentially none of its time near the center of the simplex in any of these figures. Yet it is clear that this is the one place where the population's aggregate uncertainty is low, since the population is equally suited to any environment. Instead, as time passes, the probability mass of the frequency distribution accumulates near the edges of the simplex. Here, at the edges, aggregate uncer-
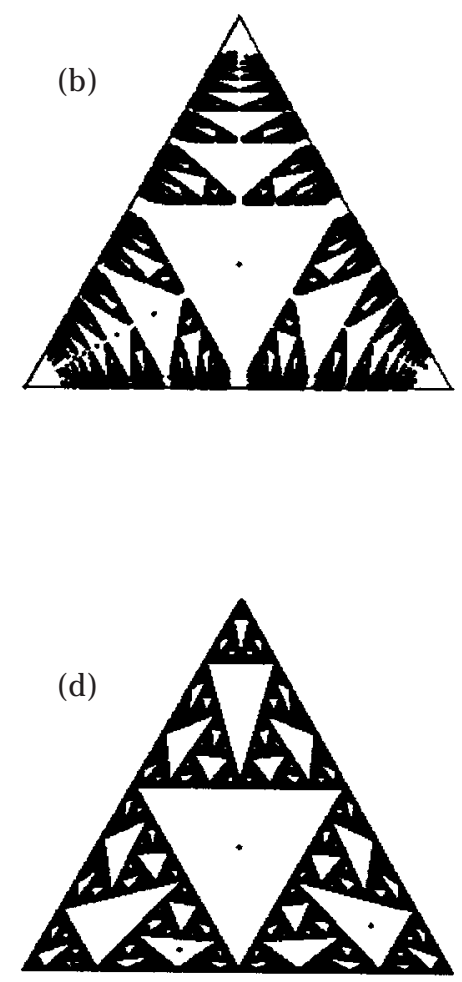

FIG. 2. Allele frequency distributions for (a) $x=y / 2$ (heterozygote advantage), (b) $x=y$ (complete dominance), (c) $x=2 y$, and (d) $x=4 y$. 
tainty is great, because at least one of the environments can devastate the population.

So what happens to long-term population growth under this model? Let us return, for the sake of illustration, to the case where $x=2 y$. Growth in each generation is a function of the environmental outcome, and the three allele frequencies $p, q$, and $r$. Suppose the allele-frequency simplex is divided into a series of regions. If in a particular generation we know the environmental outcome and the region on the simplex in which the population currently lies, we can bound the population growth in that generation.

By analogy with the previous section, consider a new, expanded set of "environmental states." Here, each state is the current environmental outcome combined with the current region in allele-frequency space. (That is, for some $k$, we characterize the environmental state by the vector of the previous $k$ environmental outcomes). We can place an upper bound on the long-term log growth rate $\rho$ for particular values of $x$ and $y=x / 2$ as follows. Split the simplex into $3^{k}$ shaded regions [as in Fig. 1(d), which shows the case $k=3$ ] yielding $3^{k+1}$ newly-defined environmental states. For each of the new environmental states, there is a maximum log-growth rate. From the results in Appendix A, if the Sierpinski Gasket has $3^{k}$ shaded triangles, then the frequency of visits to each triangle converges to $3^{-k}$. That is, after $T$ generations, as $T \rightarrow \infty$, if each triangle is visited $n(T)$ times, then with probability $1, n(T) / T \rightarrow 3^{-k}$.

To place an upper bound on $\rho$, we can use eqn (1), inserting the maximal log growth rate for each environmental state. Hence, since each environmental state arises with equal probability, $\rho$ is simply the arithmetic mean of the maximal $\log$ growth rate for each of $3^{k+1}$ environmental states. By increasing the number of shaded regions, we can obtain increasingly more stringent upper bounds. Of course, if an upper bound on $\rho$ is less than zero, population extinction is guaranteed.

Unfortunately, it is not possible to use such a method to place a lower bound on $\rho$ for the Weinshall model. This is because when there are zero-fitness entries in the fitness matrix, the log growth-rate is not bounded below in those regions which lie at the edge of the allele-frequency simplex. However, the following theorem, which uses a different line of proof (Appendix B), shows that indefinite population persistence is possible in the Weinshall model, even under conditions of hard selection.

\section{Theorem 1}

Define $\left\|z^{t}\right\|=a^{t}+b^{t}+c^{t}$. Fix $y=k x$, for any $k>0$. For any $K>0$ there exists an $L>0$ such that whenever $x>L$,

$$
\liminf _{T \rightarrow \infty} \frac{1}{T} \ln \left\|z^{T}\right\| \geqslant K .
$$

That is, the long-term log growth rate can be made arbitarily large by an appropriate choice of the homozygote fitness $x$. In particular, it is possible for an outcrossing population to survive indefinitely in the Weinshall model. This is by no means self-evident, given the results summarized in Fig. 2 in which we showed that even sexual populations tend to exhibit allele frequencies near the edges of the simplex and hence experience strikingly high levels of aggregate risk.

\subsection{CALCULATION OF LONG-TERM LOG GROWTH RATES}

While the Weinshall model does not permit the calculation of lower bounds on $\rho$ using the simpler method, there are other genetic models of evolution in random environments in which it is possible to compute both upper and lower bounds. This can be done by splitting the genotype frequency space into a finite number of regions and calculating (1) upper and lower bounds on the log-growth rate for each region, in each possible environment, and (2) calculating (or estimating by simulation) the amount of time that the population spends in each region of the genotype space. If upper and lower bounds exist for all regions, and the visitation frequency of each region converges, then one can place bounds on $\rho$. These arguments indicate an algorithm for estimating long-term log growth rates in models of genetic variation in random environments, as follows.

Suppose that the genotype frequency space is divided into a covering partition of $n$ connected, disjoint regions. Suppose furthermore that there 
are $S$ environments of which the $s$-th environment occurs independently with probability $\pi_{s}$. Let the maximum growth rate (i.e. ignoring idiosyncratic risk) in region $i$, conditional on environment $s$, be $M_{i}\left(E_{s}\right)$. Then, if the stationary probability of being in region $i$ exists for all $i$ and is given by $p_{i}$, an upper bound of $\rho$ is

$$
\rho_{u}=\sum_{i=1}^{n} \sum_{s=1}^{S} p_{i} \pi_{s} \ln \left(M_{i}\left(E_{s}\right)\right)
$$

The lower bound is calculated similarly. These upper and lower bounds on $\rho$ exist provided that upper and lower bounds on the log growth rate exist for all $i$ and $s$. (If the environments are not i.i.d., these bounds can be computed by replacing $p_{i} \pi_{s}$ with a joint probability $p_{i, s}$.)

As the number of regions $n$ is increased, in such a way that the maximum distance across each region approaches zero, the upper and lower bounds converge to a common value, provided, for example, that $\rho(p, q, r)$, the expected log growth rate conditional on the allele frequencies $p, q$ and $r$, is continuously differentiable. That is, taking $m\left(p, q, r, E_{s}\right)$ to be the expected growth rate of the population in environment $E_{s}$, conditional on allele frequencies $p, q$, and $r$, the upper and lower bounds will converge if the first partial derivatives of $m\left(p, q, r, E_{s}\right)$ with respect to $p, q$, and $r$ are all finite everywhere in allele frequency space. This result follows from an application of the Mean Value Theorem.

\section{Sex vs. Selfing}

We have shown above that the distribution of genotype frequencies in a population can affect the level of aggregate uncertainty, and hence long-term population growth and survival. It is well known that mating patterns in a population lead to different distributions of genotype frequencies: for instance selfing or positive assortative mating generally lead to heterozygote deficiencies, while self-incompatibility systems may result in excess heterozygotes. It is likely that each of these departures from a model of random-mating will impact levels of aggregate uncertainty, though the direction of the effect may in some cases be model-dependent.
In this section, we argue that the different distributions of genotype frequencies due to sex or selfing affect the level of aggregate risk encountered by a population. Thus, the differing consequences of aggregate uncertainty and idiosyncratic risk may be important in the maintenance of sexual reproduction. A wide range of explanations have been proposed for this evolutionary puzzle, including those involving the rate of evolution (Fisher, 1930; Muller, 1932), adaptation to uncertain or changing conditions (Haldane, 1932; Williams, 1975; Charlesworth, 1976), DNA repair (Bernstein \& Bernstein, 1991; Avise, 1993), avoidance of genetic deterioration (Muller, 1964; Felsenstein, 1974; Kondrashov, 1988), genetic segregation (Kirkpatrick \& Jenkins, 1989, but see also Wiener et al., 1992) and parasite resistance (Hamilton, 1980; Hamilton et al., 1990). A closely related problem, the evolution of recombination rates, has been studied in detail using modifier theory (e.g. Bergman \& Feldman, 1990, 1992; Feldman et al., 1996). A recent review of the evolution of sex problem is provided by Hurst \& Peck (1996); see also the volume by Michod \& Levin (1988).

Within the evolution of sex literature, the models most closely related to the present paper concern the effects of environmental uncertainty on population growth under sexual and asexual reproduction-namely, Moore \& Hines (1981), Hines \& Moore (1981), Weinshall (1986), Eshel \& Weinshall (1987), Weinshall \& Eshel (1987) and Roughgarden (1991). In what follows, we will turn our attention to those models, arguing that their effects are most clearly understood in the framework of idiosyncratic and aggregate risk that we have developed above.

Notice that the models treated in this paper assume that there is no random phenotypic variation (or phenotypic plasticity.) When this form of variation is present, it will have much the same effect as outcrossing in reducing aggregate uncertainty (Cooper \& Kaplan, 1982; Seger \& Brockmann, 1987). Indeed, it is plausible that asexual lineages would be under selective pressure to increase the degree of random phenotypic variation. While this strategy may be viable for some sorts of traits, it will not be viable for traits which are strongly genetically deter- 
mined-for example, immune system response to pathogens, which in mammals is largely determined by MHC type. Of course, the difference between sexual and asexual reproduction emerges only for those phenotypes which are genetically determined.

Our models also assume that there is a negligible mutation rate. In principle, an extraordinarily high mutation rate at certain critical loci could also rescue asexual lineages by regenerating new genotypes, much as outcrossing reintroduces different genotypes into sexual lineages. However, typical mutation rates appear too low to affect these conclusions. This is unsurprising; given the typical complexity of genetic traits, accelerated mutation is a strikingly inefficient mechanism for producing a few target genotypes.

As a first example of this principle, we return to Weinshall's model (see Table 1). Recall that for suitable values of the fitnesses $x$ and $y$, a random-mating sexual population is able to survive indefinitely under this model. However, as Weinshall points out, an asexual population, though it might enjoy a two-fold expected fitness advantage, is doomed to rapid extinction.

Sexuals and asexuals differ here in that each asexual genotype can be considered to be a distinct lineage. In our density-independent formulation, each lineage survives or perishes independently of all other lineages. Each lineage has zero fitness in at least one environment, and since there is probability 1 that every environment will occur within a sufficiently long time period, no lineage will survive indefinitely.

Notice that in the asexual case, genetic variation in the population plays no role in the reduction of aggregate uncertainty. Each lineage within the asexual population is genetically uniform, and suffers the full consequences of aggregate uncertainty. If, as in Weinshall's model, each lineage is separately doomed to extinction, the population itself is doomed. This argument does not hold in an outcrossing population, because such a population does not consist of genetically distinct lineages. Instead the genotypes which do poorly in one generation will be reconstituted by outcrossing (and, in multi-locus models, recombination) among the genotypes that have done well.
Though our analysis compares the growth rates of sexual and asexual types, the effect observed is not driven by group selection. Instead, it can be viewed as a model of how individual-level selection operates at a locus modifying outcrossing behavior. (For example, we might suppose that asexuality is due to a dominant allele.) Because of the mating behavior induced by this modifier, there is no gene flow between sexuals and asexuals, and hence we can take the shortcut of treating sexuals and asexuals as separate populations in order to compute the selective coefficients on the modifier locus.

While we have concentrated on two extreme cases - obligate sexuals and asexuals - in some cases neither of these may be the optimal strategy. Weinshall \& Eshel (1987) discuss the case in which individuals reproduce sexually at a rate between 0 and 1 .

For a second example of aggregate uncertainty in sexual and asexual populations, we turn to a model proposed by Roughgarden (1991); here we describe the version of the model used for Fig. 2 of that study.

Consider a population of diploid organisms, subject to a randomly chosen environment in each generation. There is one selected locus with two alleles: denoted here as A and B. In each generation, the fitness of each genotype is either $\sigma$ or $1 / \sigma$, each with probability $1 / 2$, where $\sigma>1$ is a fixed parameter. The fitness of each of the three genotypes is chosen independently of the others, in each generation. This means that there are eight equally probable environments (i.e. two possible fitnesses for each of the three genotypes). There is no serial correlation in the environment from one generation to the next.

In addition to selection, the model allows genotype frequencies to change through low levels of mutation, thereby preventing the populations from becoming monomorphic. Roughgarden uses this model to compare the performances of sexuals and asexuals. In each generation, after selection, the genotype frequencies of the sexual population return to HardyWeinberg proportions through outcrossing, while the genotype frequencies of the asexuals are left unchanged. In simulations, Roughgarden observed that sexuals consistently outperformed 
asexuals, with the magnitude of the advantage increasing with $\sigma$.

Let the frequencies of the three genotypes AA, $\mathrm{AB}$ and $\mathrm{BB}$ be denoted by $x_{1}, x_{2}$ and $x_{3}$, respectively, where $x_{1}+x_{2}+x_{3}=1$. We will now compute the expected log growth rate of the population, conditioned on the genotype frequencies, $\rho\left(x_{1}, x_{2}, x_{3}\right)$. This will give us a way of understanding how aggregate uncertainty varies across the simplex. (The idea is similar to that for the criterion $\rho(p, q, r)$ introduced at the end of the previous section.)

$$
\begin{aligned}
\rho\left(x_{1}, x_{2}, x_{3}\right) & =1 / 8\left(\log \left(\sigma x_{1}+\sigma x_{2}+\sigma x_{3}\right)\right. \\
& +\log \left(\sigma x_{1}+\sigma x_{2}+1 / \sigma x_{3}\right) \\
& +\log \left(\sigma x_{1}+1 / \sigma x_{2}+\sigma x_{3}\right) \\
& +\log \left(1 / \sigma x_{1}+\sigma x_{2}+\sigma x_{3}\right) \\
& +\log \left(\sigma x_{1}+1 / \sigma x_{2}+1 / \sigma x 3\right) \\
& +\log \left(1 / \sigma x_{1}+\sigma x_{2}+1 / \sigma x_{3}\right) \\
& +\log \left(1 / \sigma x_{1}+1 / \sigma x_{2}+\sigma x_{3}\right) \\
& \left.+\log \left(1 / \sigma x_{1}+1 / \sigma x_{2}+1 / \sigma x_{3}\right)\right) .
\end{aligned}
$$

Again, $\rho\left(x_{1}, x_{2}, x_{3}\right)$ reflects the degree of aggregate risk faced by the population at different points in genotype frequency space. When there is a stationary distribution over genotype frequencies and $\rho\left(x_{1}, x_{2}, x_{3}\right)$ is continuously differentiable, then the long-run average growth rate is given by the integral of $\rho\left(x_{1}, x_{2}, x_{3}\right)$ with respect to the stationary distribution on the simplex. In Roughgarden's model, $\rho\left(x_{1}, x_{2}, x_{3}\right)$ is continuously differentiable. Assuming only that there exists a stationary distribution over the regions in any finite partition of the simplex, as in the previous subsection, we can use $\rho\left(x_{1}, x_{2}, x_{3}\right)$ to describe the behavior of this system.

In Fig. 3 we show a contour plot of $\rho\left(x_{1}, x_{2}, x_{3}\right)$ on a De Finetti representation of the genotype space. As in the Weinshall model, the value of $\rho\left(x_{1}, x_{2}, x_{3}\right)$ depends on the location in genotype space; moreover, the highest value again occurs when the genotype frequencies are equal, at $(1 / 3$, $1 / 3,1 / 3)$. At this point, aggregate uncertainty for the population is minimized. Moving away from the center of the simplex, $\rho\left(x_{1}, x_{2}, x_{3}\right)$ becomes steadily smaller.

As Roughgarden points out, the genotype frequencies of asexuals wander around the simplex freely, while the genotype frequencies of

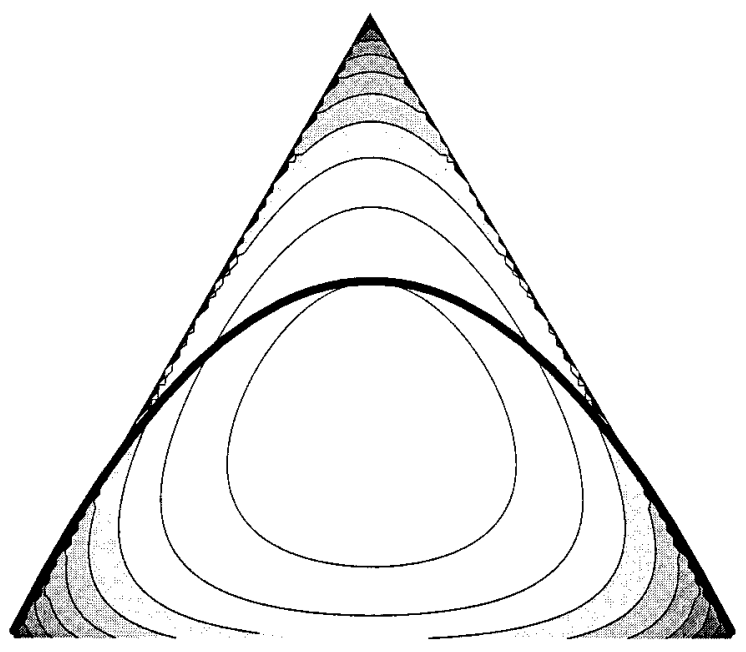

FIG. 3. Contour plot of $\rho\left(x_{1}, x_{2}, x_{3}\right)$ values on the simplex of genotype frequencies in Roughgarden's model. Lighter regions have higher $\rho\left(x_{1}, x_{2}, x_{3}\right)$ values. The Hardy-Weinberg curve is overlaid on the simplex.

the sexuals revert in each generation to the Hardy-Weinberg curve. Geometrically, return to Hardy-Weinberg proportions corresponds to strictly vertical movement on the simplex, as allele frequencies are unchanged.

From Fig. 3, it is clear that when at the top (AB) corner of the simplex, the sexual population can derive a substantial increase in its value of $\rho\left(x_{1}, x_{2}, x_{3}\right)$, by moving vertically downward to this curve. By contrast, when away from this top corner, vertical movement causes little change in the value of $\rho\left(x_{1}, x_{2}, x_{3}\right)$, as evidenced by the near-vertical contour lines in these regions. There, movement to the HardyWeinberg curve has little effect.

In this model, the highest $\rho\left(x_{1}, x_{2}, x_{3}\right)$ values are achieved not on the Hardy-Weinberg curve but rather below it [i.e. at $(1 / 3,1 / 3,1 / 3)]$. Thus, aggregate uncertainty is greater on the HardyWeinberg curve than slightly below it, where there is a heterozygote deficiency. This means that in Roughgarden's model, partial assortative mating, which generates excess homozygotes, would outperform random mating.

Hines \& Moore (1981) present a third model, closely related to those discussed above, which again shows that under some conditions there can be an advantage to outcrossing in the face of environmental fluctuations. The model is quite similar to Roughgarden's in that it deals with a 
one-locus, two-allele model of selection in a fluctuating environment. (Hines \& Moore also describe a multi-locus model which is not discussed here.) As in Roughgarden's model, the fitness of each genotype in each generation is independent of the fitnesses of the other genotypes. The difference arises from the rule used to select the fitnesses in each generation. Hines \& Moore set the heterozygote fitness to 1, and then draw the fitnesses of each of the two homozygote types independently, from normal distributions with arbitrary means and variances. As in Roughgarden (1991) and Weinshall (1986), fitnesses in a given year are independent of fitnesses in the previous years.

In exploring this model, the authors examine the change in the log ratio of sexual population size to asexual population size, conditional on the current allele frequencies in sexuals [see their eqn (2).] This corresponds to the difference in expected log growth rates of sexuals and asexuals conditional on allele frequencies. The expression that they derive for the growth of the sexuals [in their eqns (3) and (4)] is closely analogous to the measure $\rho(p, q, r)$ used in this paper for the long-term log growth rates of such populations. Moreover, since a stationary distribution exists for the allele frequencies in their model (Hines \& Moore, 1981), the arguments above can be extended to formally justify their use of this measure.

As in the models discussed above, the Hines and Moore model reveals an advantage to sexual reproduction when fitnesses fluctuate. Once again, the benefit arises from the fact that outcrossing converts some of the aggregate risk faced by a population to idiosyncratic uncertainty, thus increasing the average long-term growth rate of the population.

\section{Conclusions}

Population growth is sensitive not only to the magnitude of uncertainty in reproductive rate but also to the correlations in reproductive rate among individuals within the population. Specifically, uncertainty does less to reduce growth rates when each individual experiences reproductive success as an independent draw, than when the reproductive successes of individ- uals are correlated. We have labelled these types of risk as idiosyncratic and aggregate risk, respectively. We demonstrate that a population will grow faster if aggregate risk can be converted to idiosyncratic risk with a similar distribution, and that the presence of genetic variation can do this.

One way in which this conversion may occur is through outcrossing, and the distinction between idiosyncratic and aggregate risk appears to provide a clue to understanding the maintenance of sexual reproduction. Outcrossing serves to redistribute alleles within the population. As a consequence, the offspring of a given individual may be less correlated in phenotype, and consequently less correlated in reproductive fitness, than would be the case in an asexual population. As demonstrated in Section 2, this process provides the driving advantage to sexual reproduction in several models of outcrossing in variable environments including those presented by Weinshall (1986), Roughgarden (1991), and Hines \& Moore (1981). However, it is important to notice that our results are concerned with the evolutionary maintenance of sexual reproduction, and do not deal directly with its origins.

The ideas for this paper were conceived in a series of discussions at the Dynamic Evolutionary Game Theory Conference in Waterloo, Ontario, August 1995. The authors would like to thank I. Eshel, M. Feldman, M. Lachmann, S. Orzack, and S. Tuljapurkar for extensive comments and suggestions. A. Robson thanks the Social Sciences and Humanities Research Council of Canada for research support. C. Bergstrom and J. Pritchard were Howard Hughes Predoctoral Fellows, and were also supported by NIH grant GM 28016 to M. Feldman.

\section{REFERENCES}

Athreya, K. B. \& Ney, P. E. (1972). Branching Processes. Berlin: Springer-Verlag.

Avise, J. C. (1993). The evolutionary biology of aging, sexual reproduction, and DNA repair. Evolution 47(5), 1293-1301.

Bergman, A. \& Feldman, M. W. (1990). More on selection for and against recombination. Theor. Popul. Biol. 38, 68-92.

Bergman, A. \& Feldman, M. W. (1992). Recombination dynamics and the fitness landscape. Physica $D$ 56, 57-67.

Bernstein, C. \& Bernstein, H. (1991). Aging, Sex, and DNA Repair. New York: Academic Press. 
Charlesworth, B. (1976). Recombination modification in a fluctuating environment. Genetics 83, 181-195.

CoHEN, D. (1993). Fitness in random environments. In: Adaptation in Stochastic Environments (Yoshimura, J. \& Clark, C. W., eds) Vol. 98 of Lecture Notes in Biomathematics, pp. 8-25. New York: Springer-Verlag.

COOPER, W. S. \& KaPlan, R. H. (1982). Adaptive "coin-flipping"; a decision-theoretic examination of natural selection for random individual variation. J. theor. Biol. 94(94), 135-151.

DEMPSTER (1955). Maintenance of genetic heterogeneity. Cold Spring Harbor Symp. Quant. Biol. 20, 25-32.

Eshel, I. \& Weinshall, D. (1987). Sexual reproduction and the viability of future offspring. Am. Nat. 130(5), 775-787.

Feldman, M. W., Otto, S. P. \& Christiansen, F. B. (1996). Population genetic perspectives on the evolution of recombination.

FELSENSTEIN, J. (1974). The evolutionary advantages of recombination. Genetics $\mathbf{7 8 , 7 3 7 - 7 5 6 . ~}$

FisHer, R. A. (1930). The Genetical Theory of Natural Selection. Oxford: Oxford University Press.

Gillespie, J. (1973). Polymorphism in random environments. Theor. Popul. Biol. 4, 193-195.

GiLlespie, J. H. (1991). The Causes of Molecular Evolution. Oxford Series in Ecology and Evolution. New York: Oxford University Press.

Haccou, P. \& Iwasa, Y. (1995). Optimal mixed strategies in stochastic environments. Theor. Popul. Biol. 47(2), 212-243.

Haldane, J. B. S. (1932). The Causes of Evolution. New York: Harper.

Haldane, J. B. S. \& Jayakar, S. D. (1963). Polymorphism due to selection of varying direction. J. Genetics 58, 237-242.

Hamilton, W. D. (1980). Sex verson non-sex versus parasite. Oikos 35(2), 282-290.

Hamilton, W. D., Alexrod, R.\& Tanese, R. (1990). Sexual reproduction as an adaptation to resist parasites. Proc. Nat. Acad. Sci. 87, 3566-3573.

Hines, W. G. S. \& Moore, W. S. (1981). An analysis of sex in random environments. Adv. Appl. Prob. 13, 453-463.

Hurst, L. D. \& Peck, J. R. (1996). Recent advances in understanding of the evolution and maintenance of sex. Trends Ecol. Evol. 11(2), 46-52.

Kingman, J. F. C. (1973). Subadditive ergodic theory. Ann. Prob. 1, 883-909.

Kirkpatrick, M. \& Jenkins, C. D. (1989). Genetic segregation and the maintenance of sexual reproduction. Nature 339, 300-301.

Kondrashov, A. S. (1988). Deleterious mutations and the evolution of sexual reproduction. Nature 336, 435-440.

Kreps, D. M. (1990). A Course in Microeconomic Theory. Princeton: Princeton University Press.

Lewontin, R. C. \& Cohen, D. (1969). On population growth in a randomly varying environment. Proc. Nat. Acad. Sci. 62, 1056-1060.

Mandelbrot, B. B. (1982). The Fractal Geometry of Nature. San Francisco: W. H. Freeman.

MichoD, R. E. \& LeVIN, B. R. (1988). The Evolution of Sex. Sunderland, MA: Sinauer Associates.

Moore, W. S. \& Hines, W. G. S. (1981). Sex in random environments. J. theor. Biol. 92, 301-316.

Muller, H. J. (1932). Some genetic aspects of sex. Am. Nat. 66, 118-138.
Muller, H. J. (1964). The relation of recombination to mutational advance. Mutat. Res. 1, 2-9.

Robson, A. J. (1996). A biological basis for expected and non-expected utility. J. Econ. Theory 68, 397-424.

Roughgarden, J. (1991). The evolution of sex. Am. Nat. 138(4), 934-953.

Sasaki, A. \& Ellner, S. (1995). The evolutionarily stable phenotype distribution in a random environment. Evolution 49(2), 337-350.

SEgER \& BRockmann (1987). What is bet-hedging? Oxford Surveys Evol. Biol. 4, 182-211.

Tuljapurkar, S. D. \& OrZack, S. H. (1980). Population dynamics in variable environments I. Long-run growth rates and extinction. Theor. Popul. Biol. 18, 314-342.

Weinshall, D. (1986). Why is a two-environment system not rich enough to explain the evolution of sex? Am. Nat. 128(5), 736-750.

WeINSHALl, D. \& EshEL, I. (1987). On the evolution of an optimal rate of sexual reproduction. Am. Nat. 130(5), 758-774.

Weiner, P., Feldman, M. W. \& Otto, S. P. (1992). On genetic segregation and the evolution of sex. Evolution 46(3), 775-782.

Williams, G. C. (1975). Sex and Evolution, Vol. 8 of Monographs in Population Biology. Princeton: Princeton University Press.

Yoshimura, J. \& Clark, C. W. (1991). Individual adaptations in stochastic environments. Evol. Ecol. 5, 173-192.

\section{APPENDIX A}

\section{The Allele Frequency Distribution in a Special Case of the Weinshall Model}

Here we analyse the evolution of allele frequencies in Weinshall's model for the special case $x=2 y$. Recall from Section 2 that if allele frequencies start at an arbitrary point in the simplex, they will move to one of three possible points in the next generation. These points are halfway between the initial point and the particular vertex which is determined by the environment.

We begin by constructing a characterization of the allele frequency space in the interval $[0,1](\mathrm{M}$. Lachmann, 1996, personal communication). We represent the interval $[0,1]$ in base 4 . (We must formally distinguish between $0.033333 \ldots$ and 0.100000 , for example.) First divide the simplex into four subtriangles, as in Fig. A1, yielding the label $x_{1}$ for a given point $x$. Next divide each of the subtriangles into four smaller subtriangles, again labelling the center with 0 , the top with 1 , the left with 2 , and the right with 3 , thus yielding the next label $x_{2}$. (The subtriangles of the upside-down center subtriangle can be labelled 
arbitrarily.) Continue in this fashion dividing the simplex into further subtriangles, thereby ultimately characterizing each point $x$ in the simplex by a number in base $4, x=0 . x_{1} x_{2} x_{3} \ldots$.

If we start at a point $x=0 . x_{1} x_{2} x_{3} \ldots$, then moving halfway toward the vertex $x_{0}=1,2$, or 3 , each with probability $1 / 3$, yields the point $y=0 . x_{0} x_{1} x_{2} x_{3} \ldots$. If we start at a point $p_{0}$ on the simplex, after $n$ moves (labelled $x_{1}, x_{2}, \ldots, x_{n}$ ) we obtain the point $p^{\prime}=0 . x_{n} x_{n-1} \ldots x_{1} p_{0}$. Notice that $x_{k} x_{k-1} \ldots x_{1}$ specifies one of the $3^{k}$ shaded subtriangles of the Sierpinski triangle. Since it is equally likely that each $x_{i}=1,2$, or 3 , after at least $k$ moves there is probability $3^{-k}$ of being in each of the $3^{k}$ shaded subtriangles. Moreover, the probability distribution is clearly uniform over these shaded subtriangles.

\section{APPENDIX B}

\section{Long-term Population Growth in the Weinshall Model}

In this appendix we extend the analysis of Weinshall (1986) to the case of hard selection, in order to demonstrate that under that model, for suitably large fitnesses $x$ and $y$, a sexual population can survive indefinitely, even though all asexual types are doomed to rapid extinction. The importance of this result, in the context of this paper, is that it shows that enough genetic variation is maintained over time to keep aggregate uncertainty at manageable levels. We define genotypes, environments, and fitnesses as in Section 2.

Define $a^{t}, b^{t}$ and $c^{t}$ to be the numbers of each allele at times $t=0,1, \ldots$ and $\bar{a}^{t}=a^{t}$ $\left(a^{t}+b^{t}+c^{t}\right), \quad \bar{b}^{t} /\left(a^{t}+b^{t}+c^{t}\right) \quad$ and $\quad \bar{c}^{t}=c^{t} /$

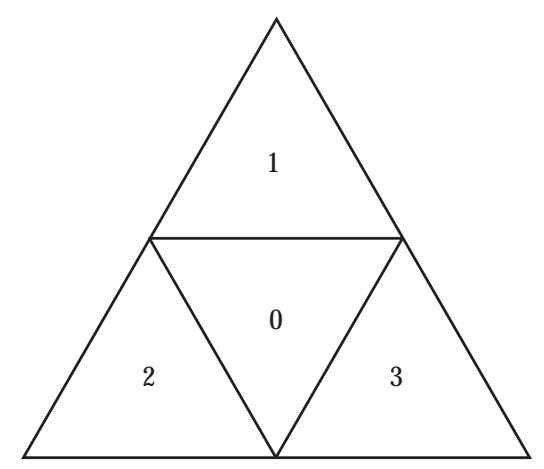

FIG. A1. Labelling the subtriangles of the simplex. $\left(a^{t}+b^{t}+c^{t}\right)$ to be the corresponding frequencies. Define matrices $m_{I}, m_{I I}$, and $m_{I I I}$ as follows:

$$
\begin{aligned}
m_{I} & =\left(\begin{array}{lll}
x & y & y \\
0 & y & 0 \\
0 & 0 & y
\end{array}\right) \\
m_{I I} & =\left(\begin{array}{lll}
y & 0 & 0 \\
y & x & y \\
0 & 0 & y
\end{array}\right) \\
m_{I I I} & =\left(\begin{array}{lll}
y & 0 & 0 \\
0 & y & 0 \\
y & y & x
\end{array}\right)
\end{aligned}
$$

Taking the allele vector at time $t$ as $\left(a^{t}, b^{t}, c^{t}\right)^{\prime}=z^{t}$, where $z^{0}$ is given, repeated application of the recursion equations (4) implies that for $T=1,2$, ... the following expression holds:

$$
z^{T}=\prod_{t \in T(I)} \bar{a}^{t} \prod_{t \in T(I I)} \bar{b}^{t} \prod_{t \in T(I I I)} \bar{c}^{t}\left(\tilde{m}^{T-1} \ldots \tilde{m}^{0} z^{0}\right)
$$

Here $\tilde{m}^{t}$ is a randomly chosen matrix, equal to $m_{i}$ if and only if the environment at date $t$ is $i=I$, $I I$, or $I I I$, and $T(i)$ is the subset of $\{0, \ldots, T-1\}$ for which the environment is $i=I, I I, I I I$, where the norm $\|\cdot\|$ denotes the sum of the entries of a vector or matrix with all non-negative entries. Then the total number of alleles at date $T$ is

$$
\begin{aligned}
& \left\|z^{T}\right\|=a^{T}+b^{T}+c^{T} \\
& \quad=\prod_{t \in T(I)} \bar{a}^{t} \prod_{t \in T(I I)} \bar{b}^{t} \prod_{t \in T(I I)} \bar{c}^{t}\left\|\tilde{m}^{T-1} \ldots \tilde{m}^{0} z^{0}\right\|
\end{aligned}
$$

The subadditive ergodic theorem applied to random matrices with non-negative elements (see Kingman, 1973) implies that,

$$
\begin{aligned}
\lim _{T \rightarrow \infty} & \frac{1}{T} \ln \left\|\tilde{m}^{T-1} \ldots \tilde{m}^{0} z^{0}\right\| \\
\quad & \lim _{T \rightarrow \infty} \frac{1}{T} \ln \left\|^{T-1} \ldots \tilde{m}^{0}\right\|=\rho_{1}<\infty
\end{aligned}
$$

say, as $T \rightarrow \infty$, independently of $z^{0}=$ $\left(a^{0}, b^{0}, c^{0}\right) \gg(0,0,0)$. That is, this limit exists and is also independent of the sequence of environments, with probability 1 . Note that all matrices here have finite elements. Consider this theorem 
applied to the case where the matrices $m_{I}, m_{I I}$ and $m_{I I I}$ are replaced by

$$
\begin{aligned}
& \tilde{m}_{1}=\left(\begin{array}{lll}
x & 0 & 0 \\
0 & y & 0 \\
0 & 0 & y
\end{array}\right) \\
& \tilde{m}_{2}=\left(\begin{array}{lll}
y & 0 & 0 \\
0 & x & 0 \\
0 & 0 & y
\end{array}\right) \\
& \tilde{m}_{3}=\left(\begin{array}{lll}
y & 0 & 0 \\
0 & y & 0 \\
0 & 0 & x
\end{array}\right)
\end{aligned}
$$

respectively. It follows that

$$
\rho_{1} \geqslant(1 / 3) \ln x+(2 / 3) \ln y>-\infty,
$$

so that $\rho_{1}$ can be chosen as large as desired by choosing $x$ and $y$ large enough. The growth of the total number of allele copies, as reflected in the behavior of the expression $1 / T \ln \left\|z^{T}\right\|$ as $T \rightarrow \infty$, then depends on the behavior of

$$
\frac{1}{T} \ln \left(\prod_{t \in T(I)} \bar{a}^{t} \prod_{t \in T(I I)} \bar{b}^{t} \prod_{t \in T(I I I)} \bar{c}^{t}\right),
$$

as $T \rightarrow \infty$. Of course, the above term is no less than

$$
\frac{1}{T} \ln \left(\prod_{t=0}^{T-1} \bar{a}^{t}\right)+\frac{1}{T} \ln \left(\prod_{t=0}^{T-1} \bar{b}^{t}\right)+\frac{1}{T} \ln \left(\prod_{t=0}^{T-1} \bar{c}^{t}\right) .
$$

Consider then the behavior of

$$
\frac{1}{T} \ln \left(\prod_{t=0}^{T-1} \bar{a}^{t}\right)=\frac{1}{T} \sum_{t=0}^{T-1} \ln \bar{a}^{t}
$$

as $T \rightarrow \infty$, since the behavior of the other two terms must be similar. Introducing appropriate time superscripts to eqns (4), it follows that, if the environment at time $t$ is $\mathrm{I}$,

$\bar{a}^{t-1}=\bar{a}_{I}^{t+1}=\frac{\bar{a}^{t} x+\bar{b}^{t} y+\bar{c}^{t} y}{\bar{a}^{t} x+2 b^{t} y+2 \bar{c}^{t} y} \geq 1 / 2$,

regardless of $x, y>0$. (i) Consider first the case that $2 y \geqslant x$. If the environment at time $t$ is II or III, then

$$
\begin{aligned}
& \bar{a}^{t+1}=\bar{a}_{I I}^{t+1}=\frac{\bar{a}^{t} y}{2 \bar{a}^{t} y+\bar{b}^{t} x+2 \bar{c}^{t} y} \geq \bar{a}^{t} / 2 \text { or } \\
& \bar{a}^{t+1}=\bar{a}_{I I I}^{t+1}=\frac{\bar{a}^{t} y}{2 \bar{a}^{t} y+2 b^{t} x+\bar{c}^{t} y} \geq \bar{a}^{t} / 2 .
\end{aligned}
$$

It can be shown to follow that

$$
\frac{1}{T} \sum_{t=0}^{T-1} \ln \bar{a}^{t} \geq \frac{\tilde{t}+1}{T} \ln 2 \bar{a}^{0}-\frac{1}{T} \sum_{t=0}^{T-1} \tilde{\mathrm{e}}^{t} \ln 2,
$$

where $\tilde{t}$ is a random variable representing the time of the first appearance of environment $I$. (It is enough to consider only the case that $T \geqslant \tilde{t}+1$, since the limit as $T \rightarrow \infty$ will be taken and $\tilde{t}$ is finite with probability one.) In addition, $\tilde{\mathrm{e}}^{t}$ is the associated random variable defined by

$$
\mathrm{e}^{0}=1, \tilde{\mathrm{e}}^{t+1}=\left\{\begin{array}{cc}
\mathrm{e}^{t}+1, & \text { with probability } 2 / 3 \\
1 & \text { with probability } 1 / 3
\end{array}\right\},
$$

$$
\text { independently at } t=0,1, \ldots \text {. }
$$

It is not hard to show that, with probability 1 ,

$$
\frac{\tilde{t}+1}{T} \rightarrow 0
$$

and

$$
\frac{1}{T} \prod_{t=0}^{T-1} \tilde{\mathrm{e}}^{t} \rightarrow(1 / 6) E((\tilde{t}+1)(\tilde{t}+2)),
$$

as $T \rightarrow \infty$. Now

$$
\begin{array}{r}
E(\tilde{t}+1)(\tilde{t}+2))=(1 / 3) 2+\ldots+ \\
(2 / 3)^{k}(1 / 3)(k+1)(k+2)+\ldots \equiv L<\infty,
\end{array}
$$

by the ratio test. Where $\underline{\lim }$ denotes the limit inferior of a sequence, it follows that

$\underline{\lim }_{T \rightarrow \infty} \frac{1}{T} \sum_{t=0}^{T-1} \ln \bar{a}^{t} \geq-(L / 6) \ln 2>-\infty$

with probability 1 , so

$$
\begin{array}{r}
\underline{\lim }_{T \rightarrow \infty} \frac{1}{T} \ln \left(\prod_{t \in T(I)} \bar{a}^{t} \prod_{t \in T(I I)} \bar{b}^{t} \prod_{t \in T(I I)} \bar{c}^{t}\right) \\
\geq-(L / 2) \ln 2>-\infty,
\end{array}
$$


with probability 1 . Finally then, recalling eqns (B.3) and (B.6),

$$
\begin{aligned}
\underline{\lim }_{T \rightarrow \infty} \frac{1}{T} \ln \left\|z_{T}\right\| & \geq(1 / 3) \ln x \\
& +(2 / 3) \ln y-(L / 2) \ln 2,
\end{aligned}
$$

with probability 1 . It follows that the sexual population is essentially bounded below by a population growing at a rate which can be made as large as desired by choosing large enough $x$ and $y$. In particular, then, the sexual population is not condemned to asymptotic decline.

(ii) Consider now the case that $x \geqslant 2 y$. If the environment at time $t$ is II or III,

$\bar{a}^{t+1}=\bar{a}_{I I}^{t+1}=\frac{\bar{a}^{t} y}{2 \bar{a}^{t} y+\bar{b}^{t} x+2 \bar{c}^{t} y} \geq \bar{a}^{t} y / x$

or

$\bar{a}^{t+1}=\bar{a}_{I I I}^{t+1}=\frac{\bar{a}^{t} y}{2 \bar{a}^{t} y+2 b^{t} x+\bar{c}^{t} y} \geq \bar{a}^{t} y / x$.
It follows readily that

$$
\frac{1}{T} \sum_{t=0}^{T-1} \ln \bar{a}^{t} \geq \frac{\tilde{t}+1}{T} \ln \frac{\bar{a} x}{y}-\frac{1}{T} \sum_{t=0}^{T-1} \tilde{\mathrm{e}}^{t} \ln (x / y),
$$

where $\tilde{t}$ and $\tilde{\mathrm{e}}^{t}$ are as defined above for $\tilde{t}+1 \leqslant T$. Making additional minor changes in the previous argument, it follows that

$$
\begin{aligned}
\underline{\lim }_{t \rightarrow \infty} & \frac{1}{T} \ln \left\|z^{T}\right\| \geq(1 / 3) \ln x \\
& +(2 / 3) \ln y-(L / 2) \ln (x / y),
\end{aligned}
$$

with probability 1 , so it is still true that the sexual population is essentially bounded below by a population growing at an exponential rate which can be made as large as desired by choosing suitable large $x$ and $y$. Again, the sexual population is not bound to decline to 0 , in particular. Therefore, it is clear that a sexual population can outcompete an asexual population, despite a two-fold cost of sex, simply because the asexual population is bound to go extinct while the sexual population will persist. 